\title{
Центральный гипотиреоз
}

\author{
Н.А. Петунина, А.В. Трухина, Н.С. Мартиросян \\ ФГАОУ ВО «Первый Московский государственный медицинский университет им. И.М. Сеченова» Минздрава России (Сеченовский \\ Университет), Москва, Россия
}

\begin{abstract}
Аннотация
Центральный гипотиреоз является реАкой формой гипотиреоза, обусловленной поражением гипофиза (вторичный гипотиреоз) или гипоталамуса (третичный гипотиреоз). Затруднения в Аиагностике и ведении больных обусловлены стертой к^инической картиной,

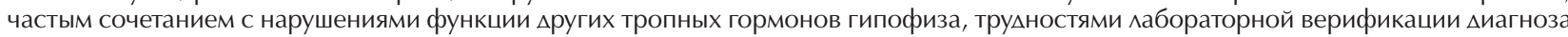
при высоком уровне тиреотропного гормона (ТТГ) и низком и^и норма^ьном уровне свободного Т4. Аиагностика основывается на подтвержденном снижении уровня свободного Т4 при низком или нормальном уровне ТТГ. Стандартом ^ечения гипотиреоза ^юбой этиологии при этом остается монотерапия ^евотироксином натрия, позволяюшая восстановить эутиреоидное состояние у большинства пациентов. Критерием эффективности терапии является подцержание уровня свободного Т4 в верхней половине референсного интервала нормы. В статье приведены современные представления об эпидемиологии, патогенезе и стратегии ведения пациентов с центральным гипотиреозом.
\end{abstract}

КАючевые слова: центральный гипотиреоз, гипопитуитаризм, L-тироксин.

А^я цитирования: Петунина Н.А., Трухина А.В., Мартиросян Н.С. Центральный гипотиреоз: современные представления о диагностике и лечении. Терапевтический архив. 2019; 91 (10): 135-138. DOI: 10.26442/00403660.2019.10.000358

\section{Central hypothyroidism}

\section{N.A. Petunina, L.V. Trukhina, N.S. Martirosian}

Sechenov First Moscow State Medical University (Sechenov University), Moscow, Russia

\begin{abstract}
Central hypothyroidism is a rare cause of hypothyroidism, consequence of various disorders affecting pituitary (secondary) or hypothalamus (tertiary hypothyroidism). Difficulties in the diagnosis and management of patients are due to the nontypical clinical picture, frequent combination with impaired function of other pituitary hormones, difficulties in laboratory assessment in high TSH levels or low-normal T4 free levels. Diagnosis is based on a confirmed decrease in the level of free T4 with a low or normal level of $\mathrm{TSH}$. The standard treatment for hypothyroidism of any etiology remains monotherapy with levothyroxine, which allows to restore the euthyroid state in most patients. The criterion for the effectiveness of therapy is to maintain the level of T4 free in the upper half of the reference norm interval. The article presents a modern understanding of epidemiology, pathogenesis and strategies for managing patients with central hypothyroidism.
\end{abstract}

Keywords: central hypothyroidism, hypopituitarism, L-thyroxine.

For citation: Petunina N.A., Trukhina L.V., Martirosian N.S. Central hypothyroidism: modern conceps in diagnostics and treatment. Therapeutic Archive. 2019; 91 (10): 135-138. DOI: 10.26442/00403660.2019.10.000358

АКТГ - адренокортикотропный гормон
ТРГ - тиреотропин-рилизинг гормон
ТТГ - тиреотропный гормон
ЩЖ - щитовидная железа

ЕТА - Европейская тиреоидологическая ассоциация

\section{Введение}

Гипотиреоз представляет собой клинический синдром, обусловленный недостатком тиреоидных гормонов в сыворотке крови или стойким снижением действия гормонов на тканимишени. Наиболее частой причиной гипотиреоза, на долю которой приходится до $99 \%$ всех случаев, является первичный гипотиреоз, обусловленный недостаточным синтезом тиреоидных гормонов щитовидной железой (ЩЖ). Центральный гипотиреоз встречается реже, он связан с поражением гипофиза (вторичный гипотиреоз) и/или гипоталамуса (третичный гипотиреоз) и характеризуется нарушением синтеза тиреоидных гормонов вследствие недостаточности стимулирующего действия тиреотропного гормона (ТТГ) на неизмененную ЩЖ. Дифференциальная диагностика вторичного и третичного гипотиреоза в клинической практике представляет значительную сложность, в связи с чем их часто объединяют термином «центральный (гипоталамо-гипофизарный) гипотиреоз». Центральный гипотиреоз встречается в любом возрасте; в отличие от первичного гипотиреоза, для заболеваемости не характерны гендерные различия. Прева- лентность центрального гипотиреоза в общей популяции составляет около 1:80 000 - 1:120 000 [1]. Среди новорожденных с врожденным гипотиреозом один из 30-40 случаев обусловлен центральным гипотиреозом. Это гетерогенная патология, которая может быть обусловлена как различными приобретенными заболеваниями гипоталамо-гипофизарной системы, так и врожденными или спорадическими генетическими дефектами, приводящими к нарушению синтеза тиреотропин-рилизинг гормона (ТРГ) или ТТГ. В некоторых случаях встречается изолированный дефицит тропной функции ТТГ, однако в большинстве случаев речь идет о дефиците нескольких тропных гормонов в рамках частичного или тотального гипопитуитаризма. Зачастую гипотиреоз носит нетяжелое течение, а трудности диагностики обусловлены стертостью клинической картины вследствие сопутствующей патологии гипоталамо-гипофизарной системы. До недавнего времени отсутствовали единые подходы в ведении данной категории больных, однако в 2018 г. эксперты Европейской тиреоидологической ассоциации (ЕТА) обобщили накопленный опыт, представив клинические рекомендации по диагностике и лечению центрального гипотиреоза [2]. 


\section{Этиомогия и патогенез}

Наиболее частыми причинами центрального гипотиреоза у новорожденных и младенцев являются врожденная патология гипоталамо-гипофизарной системы, характеризующаяся гипоплазией гипофиза, и генетические мутации, приводящие к изолированному центральному гипотиреозу (мутация $T S H B)$ [3]. Ряд генетических аномалий в свою очередь могут быть причиной манифестации центрального гипотиреоза и в более позднем возрасте. Гипоплазия гипофиза обычно клинически протекает с недостаточностью или отсутствием секреции ТТГ и адренокортикотропного гормона (АКТГ). У взрослых наиболее частыми причинами приобретенного центрального гипотиреоза являются аденомы и другие образования гипофиза. Рост аденомы гипофиза может привести к компрессии тиреотрофов, нарушению гипоталамо-гипофизарного кровотока или, редко, острому кровоизлиянию или инфаркту с апоплексией гипофиза. Другими причинами приобретенного центрального гипотиреоза являются поражения гипоталамо-гипофизарной области вследствие травмы, облучения или хирургического вмешательства, развития инфекционных и аутоиммунных заболеваний. При нарушении функции аденогипофиза чаще всего имеет место недостаточная секреция двух или более тропных гормонов гипофиза вплоть до пангипопитуитаризма. Наиболее распространены сочетание вторичного гипотиреоза и вторичного гипокортицизма, что обязательно необходимо учитывать при определении тактики лечения таких паициентов. Причины центрального гипотиреоза приведены в таблице.

Патогенетические механизмы развития центрального гипотиреоза могут быть обусловлены несколькими факторами: снижением количества ТТГ-продуцирующих тиреотрофов и уровня ТТГ (снижением резерва ТТГ); секрецией биологически неактивных иммунореактивных форм ТТГ, не способных стимулировать рецептор ТТГ, однако обусловливающих парадоксально высокий уровень ТТГ при центральном гипотиреозе; нарушением стимуляции синтеза ТТГ или функционирования гипоталамо-гипофизарно-тиреоидной оси и принципа положительной и отрицательной обратной связи с ЩЖ (резистентность тиреотропин-рилизинг гормона, мутация $T B L 1 X)[4,5]$.

\section{Аиагностика}

Клиническая картина гипотиреоза любой этиологии сходна, неспецифична и обусловлена снижением периферического уровня тиреоидных гормонов. Клинические проявления центрального гипотиреоза зависят от этиологии, сопутствующей недостаточности других тропных гормонов и возраста на момент манифестации заболевания. Существуют некоторые особенности течения центрального гипотиреоза по сравнению с первичным. Обменно-гипотермический синдром может протекать без избытка массы тела и ожирения или гиперхолестеринемии. Менее выражен при центральном гипотиреозе отечный синдром, не характерны недостаточность кровообращения, гипотиреоидный полисерозит, гепатомегалия, анемический синдром. Зачастую центральный гипотиреоз характеризуется легким или умеренной тяжести течением, поскольку редко сопровождается полным истощением резерва

\section{Сведения об авторах:}

Петунина Нина Александровна - д.м.н., проф., зав. каф. эндокринологии лечебного факультета; ORCID: 0000-0001-9390-1200

Трухина Любовь Валентиновна - к.м.н., доц. каф. эндокринологии лечебного факультета; ORCID: 0000-0001-8997-4984

\section{Причины центрахьного гипотиреоза}

\begin{tabular}{ll}
\hline $\begin{array}{l}\text { Основные группы } \\
\text { причин }\end{array}$ & \multicolumn{1}{c}{ Этиология } \\
\hline Образования & Макроаденома гипофиза \\
гипофиза & Краниофарингиома \\
& Менингиома \\
& Глиома \\
& Киста кармана Ратке \\
& Метастазы злокачестенных \\
& опухолей другой локализации \\
(рак легкого, молочной железы и др.) \\
Аневризма внутренней сонной артерии \\
Ятрогенные & Операции на гипофизе/облучение \\
причины & Прием лекарственных препаратов \\
Травмы & митотан, бексаротен и др.) \\
Острые & Травма головы \\
сосудистые & Инфаркт гипофиза \\
расстройста & Синдром Шихана \\
Аутоиммунные & Субарахноидальные кровоизлияния \\
заболевания & Послеродовый гипофизит \\
Инфильтративные & Лимфоцитарный гипофизит \\
заболевания & Синдром перегрузки железом \\
Иаркоидоз \\
Инфекционные & Гистиоцитоз Х \\
заболевания & Туберкулез \\
Врожденные & Микозы \\
дефекты & Сифилис \\
& Гипопитуитаризм или \\
изолированный центральный \\
гипотиреоз \\
\end{tabular}

ТТГ. Легче протекает приобретенный гипотиреоз, наиболее тяжело - врожденный гипотиреоз, обусловленный мутацией гена TSH $\beta$ [6]. Кроме того, клиническая картина маскируется сопутствующей патологией гипоталамо-гипофизарной системы, неврологической симптоматикой и/или аденогипофизарной недостаточностью (недостаток соматотропного гормона, вторичный гипогонадизм, вторичный гипокортицизм), несахарным диабетом, гиперпролактинемией.

Учитывая неспецифичность клинической картины и потенциальные последствия нелеченого гипотиреоза, эксперты ETA рекомендуют скрининг на центральный гипотиреоз в следующих группах [2]:

- у детей с семейным анамнезом центрального гипотиреоза, задержкой развития, отставанием роста, недостаточностью гормона роста, с отставанием либо преждевременным половым созреванием или другими признаками патологии гипоталамо-гипофизарной системы;

- при наличии персонального или семейного анамнеза образований гипоталамо-гипофизарной области;

- при тяжелых черепно-мозговых травмах;

- при остром нарушении мозгового кровообращения/инсульте;

- при облучении головы;

- при гемохроматозе или синдроме перегрузки железом;

Контактная информация:

Мартиросян Нарине Степановна - к.м.н., ассистент каф. эндокринологии лечебного факультета; тел.: +7(926)275-05-25; e-mail: narinarine@list.ru; ORCID: 0000-0002-0202-1257 
- у пациентов, у которых клиническая картина гипотиреоза сочетается с клиническими признаками других гипоталамо-гипофизарных заболеваний (гиперпролактинемии, акромегалии, несахарного диабета, головными болями, нарушениями полей зрения).

Кроме того, манифестацию центрального гипотиреоза необходимо исключить после начала терапии препаратами гормона роста или эстрогенами у пациентов с вторичным гипогонадизмом или недостаточностью соматотропного гормона, а также у пациентов, получающих терапию агонистами RXR-рецепторов (бексаротен), ингибиторами CTLA-4, PD-1, PD-L1 (ипилимумаб и др.) или митотаном (показан токсический эффект на тиреотрофы) [7-9].

В отличие от первичного гипотиреоза, лабораторная диагностика основывается на определении двух тестов - уровня ТТГ и свободного Т4. В типичном случае для центрального гипотиреоза характерен сниженный уровень ТТГ и свободного Т4. Однако у некоторых больных с вторичным гипотиреозом уровень ТТГ в крови либо находится в пределах нормы, либо парадоксально повышен, что связывают с секрецией иммунореактивного, но биологически неактивного ТТГ. Несоответствие клинической картины, степени снижения свободного Т4 уровню ТТГ, который обычно не превышает 10 мЕд/л, позволяет заподозрить вторичный гипотиреоз.

Диагноз необходимо подтвердить повторной оценкой ТТГ и свободного Т4 при исключении факторов, которые могут повлиять на интерпретацию результатов. Изолированное снижение свободного ТЗ требует исключения синдрома эутиреоидной патологии, изолированная гипотироксинемия может наблюдаться в I триместре беременности, ряд лекарственных препаратов могут также оказывать временный ТТГ-супрессивный эффект (глюкокортикоиды, противоэпилептические препараты, соматостатин) [7]. В типичных случаях повторное определение низких уровней свободного Т4 и ТТГ позволяет подтвердить манифестный гипотиреоз. В случаях легкого гипотиреоза с низконормальным/пограничным уровнем Т4 диагностика центрального гипотиреоза затруднена, при этом даже легкий гипотиреоз может быть причиной снижения умственного развития и замедления скорости роста у детей. У пациентов, наблюдающихся по поводу патологии гипоталамо-гипофизарной области, рекомендуется ежегодная оценка ТТГ и свободного Т4, легкий центральный гипотиреоз необходимо заподозрить при снижении уровня свободного Т4 с верхненормального уровня в нижнюю квартиль референсного значения нормы или более чем на $20 \%$ по сравнению с предыдущим исследованием (при условии определения в одной лаборатории той же методикой) $[2,10]$. В пользу манифестации центрального гипотиреоза также будет говорить наличие недостаточности других тропных гормонов. Для диагностики центрального гипотиреоза возможно проведение пробы с тиролиберином, в ходе которой исследуют уровень ТТГ исходно и через 30 мин после внутривенного введения 200 мкг тиролиберина. При первичном гипотиреозе после введения тиролиберина уровень ТТГ поднимается выше $25 \mathrm{mE} / л$, при вторичном - не изменяется.

В подавляющем большинстве случаев центральный гипотиреоз сочетается с недостаточностью других тропных функций аденогипофиза, т. е. встречается при гипопитуитаризме. В этой ситуации особенные усилия необходимо направить на исключение наличия надпочечниковой недостаточности. Вторичный гипотиреоз в рамках гипофизарной недостаточности необходимо дифференцировать от аутоиммунных полигландулярных синдромов, при которых также имеется недостаточность сразу нескольких эндокринных желез (ЩЖ, кора надпочечников, гонады). Наиболее часто встречается аутоиммунный полигландулярный синдром типа 2, представленный сочета- нием надпочечниковой недостаточности аутоиммунного типа с аутоиммунным тиреоидитом (синдром Шмидта) и/или сахарным диабетом 1-го типа (синдром Карпентера). Дифференциальная диагностика в этом случае основывается на определении уровня тропных гормонов гипофиза.

\section{Аечение}

Основным принципом лечения синдрома гипотиреоза является замещение функции ЩЖ препаратами тиреоидных гормонов. Целью заместительной терапии гипотиреоза является купирование симптомов гипотиреоза, восстановление эутиреоза и предупреждение передозировки с развитием ятрогенного тиреотоксикоза. Лечение центрального гипотиреоза должно быть начато после исключения надпочечниковой недостаточности или начала заместительной терапии глюкокортикоидами у пациентов с вторичной надпочечниковой недостаточностью или в случаях, когда таковую невозможно исключить для профилактики адреналового криза. Так же как и при первичном гипотиреозе, левотироксин является препаратом выбора для лечения центрального гипотиреоза. Единственное контролируемое клиническое исследование при центральном гипотиреозе не показало преимуществ комбинированной терапии левотироксином и трийодтиронином [11]. Кроме того, учитывая невозможность использования для оценки эффективности лечения уровня ТТГ, риск передозировки комбинированного препарата и ятрогенного тиреотоксикоза намного выше, чем при первичном гипотиреозе. Таким образом, «золотым стандартом» терапии гипотиреоза любой этиологии остается левотироксин. Препарат имеет высокую биодоступность (около 70-80\%), длительный период полужизни (около 7 дней), что позволяет принимать его один раз в сутки, поддерживая стабильный уровень Т4 и Т3 сыворотки крови. Данная терапия характеризуется высокой эффективностью в отношении регресса симптомов гипотиреоза у большинства пациентов, низкой стоимостью и хорошим профилем безопасности. Особенности фармакокинетики обусловливают необходимость принимать препарат натощак, желательно утром, за 30-40 мин до приема пищи и других препаратов [12]. Однако при невозможности приема левотироксина утром препарат может быть назначен перед сном, но не ранее чем через 4 ч после приема пищи [13]. Доза левотироксина определяется возрастом пациента, массой тела и наличием сопутствующих сердечно-сосудистых заболеваний. Взрослым пациентам с центральным гипотиреозом, молодого возраста, без тяжелой сердечно-сосудистой патологии рекомендовано назначение левотироксина в стартовой дозе 1,2-1,6 мкг/сут. Учитывая положительное инотропное и хронотропное действие тиреоидных гормонов на миокард, что повышает потребность миокарда в кислороде, и связанные с этим риски развития ишемии миокарда и аритмий при назначении первоначально больших доз левотироксина, пациентам пожилого возраста, а также лицам с осложненным анамнезом по сердечно-сосудистым заболеваниям рекомендуется назначение меньшей стартовой дозы левотироксина - 25 мкг, с последующей титрацией дозы препарата каждые 6-8 нед до достижения эутиреоза [14]. Как и при первичном гипотиреозе, лечение легких форм центрального гипотиреоза у пациентов старше 75 лет нецелесообразно, поскольку возможные риски превышают пользу.

Мониторинг эффективности терапии центрального гипотиреоза рекомендовано проводить по уровню свободных тиреоидных гормонов, при этом забор крови должен быть осуществлен до или как минимум через 4 ч после приема $\mathrm{L}$-тироксина. После назначения стартовой дозы рекомендуется контроль свободного Т4 и ТТГ через 6-8 нед для оценки адекватности дозировки [2]. Ряд авторов рекомендуют 
поддерживать целевой уровень свободного Т4 в верхней части референсного интервала $[11,15,16]$. Эксперты ЕТА рекомендуют поддерживать целевой уровень свободного Т4 выше среднего значения референсного интервала нормы [2]. У пациентов с исходно нормальным или повышенным уровнем ТТГ на фоне заместительной терапии отмечается подавление ТТГ, что также косвенно может отражать эффективность проводимой терапии, в свою очередь нормальный ТТГ в данном случае будет свидетельствовать о недостаточной дозе левотироксина. Недостаточность дозы левотироксина необходимо заподозрить при низконормальном или низком свободном Т4, особенно при повышении уровня ТТГ $>1$ мЕд/л [2]. Необходимо помнить, что доза левотироксина может зависеть от сопутствующего вторичного гипогонадизма и необходимости в заместительной терапии эстрогенами. Потребность в левотироксине также может меняться при подготовке пациенток к беременности с помощью вспомогательных репродуктивных технологий.

Повышение дозы левотироксина может потребоваться в случаях начала терапии гормонами роста, эстрогенами или оральными контрацептивами, стимуляции яичников, при повышении массы тела, развитии пубертата, приеме препаратов, влияющих на всасывание и метаболизм тироксина. В период беременности потребность в тиреоидных гормонах возрастает на 30-50\%, что необходимо учитывать при назначении левотироксина или коррекции его дозы. С возрастом потребность в левотироксине постепенно снижается, что связано с замедлением клиренса тироксина и снижением мышечной массы, снижение дозы левотироксина также может потребоваться при переходе в менопаузу и значительном снижении массы тела. Передозировка левотироксина, кроме потенциальной опасности усугубления течения ИБС, может приводить и к другим осложнениям уменьшению минеральной плотности костной ткани и по- вышению риска переломов, развитию фибрилляции предсердий. После изменения дозы левотироксина повторную оценку ТТГ и Т4 необходимо провести через 4-6 нед. При достижении стабильной заместительной дозы левотироксина эксперты ETA рекомендуют ежегодную оценку свободного Т4, а уровни ТТГ и свободного Т3 должны оцениваться только при подозрении на передозировку или недостаточность дозы левотироксина [2].

Причинами отсутствия компенсации гипотиреоза могут быть неадекватная доза левотироксина, плохая приверженность пациента к лечению, взаимодействие с сопутствующими препаратами, прием левотироксина вместе с пищей, синдром мальабсорбции, сопутствующий аутоиммунный гастрит, целиакия и другие заболевания, влияющие на всасывание препарата.

\section{ЗакАючение}

Центральный гипотиреоз представляет собой сложную гетерогенную проблему. Лучшее понимание патогенеза заболевания в будущем может предложить инструменты для более точной и ранней диагностики центрального гипотиреоза, особенно легких форм, и определения индивидуального целевого уровня свободного Т4. Сегодня диагностика основывается в первую очередь на определении уровня ТТГ и свободного Т4 в группах риска развития центрального гипотиреоза, куда входят все больше пациентов, получающих новые таргетные иммуносупрессивные препараты. Назначение адекватных доз левотироксина, поддерживающих уровень свободного Т4 выше среднего референсного значения, позволяет компенсировать клинические проявления гипотиреоза и улучшить качество жизни пациентов.

Авторы заявляют об отсутствии конфликта интересов.

\section{АИТЕРATYPA/REFERENCES}

1. Lania A, Persani L, Beck-Peccoz P. Central hypothyroidism. Pituitary. 2008;11(2):181-6. doi: 10.1007/s11102-008-0122-6

2. Persani L, Brabant G, Dattani M, Bonomi M, Feldt-Rasmussen U, Fliers E, Gruters A, Maiter D, Schoenmakers N, van Trotsenburg ASP. 2018 European Thyroid Association (ETA) Guidelines on the Diagnosis and Management of Central Hypothyroidism. Eur Thyroid $J$. 2018;7:225-37. doi: 10.1159/000491388

3. Beck-Peccoz P, Rodari G, Giavoli C, Lania A. Central hypothyroidism a neglected thyroid disorder. Nat Rev Endocrinol. 2017;Oct;13(10):588 98. doi: 10.1038/nrendo.2017.47

4. Heinen CA, Losekoot M, Sun Y, Watson PJ, Fairall L, Joustra SD, Zwaveling-Soonawala N, Oostdijk W, van den Akker EL, Alders M, Santen GW, van Rijn RR, Dreschler WA, Surovtseva OV, Biermasz NR, Hennekam RC, Wit JM, Schwabe JW, Boelen A, Fliers E, van Trotsenburg AS. Mutations in TBL1X are associated with central hypothyroidism. J Clin Endocrinol Metab. 2016;101:4564-73. doi: 10.1210/jc.2016-2531

5. Faglia G, Bitensky L, Pinchera A, Ferrari C, Paracchi A, Beck-Peccoz P, Ambrosi B, Spada A. Thyrotropin secretion in patients with central hypothyroidism: evidence for reduced biological activity of immunoreactive thyrotropin. J Clin Endocrinol Metab. 1979;48:989-98.

6. García M, González de Buitrago J, Jiménez-Rosés M, Pardo L, Hinkle PM, Moreno JC. Central Hypothyroidism Due to a TRHR Mutation Causing Impaired Ligand Affinity and Transactivation of Gq. J Clin Endocrinol Metab. 2017 Jul 1;102(7):2433-42. doi: 10.1210/jc.2016-3977

7. Persani L. Clinical review: Central hypothyroidism: pathogenic, diagnostic, and therapeutic challenges. J Clin Endocrinol Metab. 2012 Sep;97(9):3068-78. doi: 10.1210/jc.2012-1616

8. Haugen BR. Drugs that suppress TSH or cause central hypothyroidism Best Pract Res Clin Endocrinol Metab. 2009 Dec;23(6):793-800. doi: 10.1016/j.beem.2009.08.003

9. Russo M, Scollo C, Pellegriti G, Cotta OR, Squatrito S, Frasca F, Cannavò $\mathrm{S}$, Gullo D. Mitotane treatment in patients with adrenocortica

cancer causes central hypothyroidism. Clin Endocrinol (Oxf). 2016 Apr;84(4):614-9. doi: 10.1111/cen.12868

10. Alexopoulou O, Beguin C, De Nayer P, Maiter D. Clinical and hormonal characteristics of central hypothyroidism at diagnosis and during follow-up in adult patients. Eur J Endocrinol. 2004 Jan;150(1):1-8.

11. Slawik M, Klawitter B, Meiser E, Schories M, Zwermann O, Borm K, Peper M, Lubrich B, Hug MJ, Nauck M, Olschewski M, Beuschlein F, Reincke M. Thyroid hormone replacement for central hypothyroidism: a randomized controlled trial comparing two doses of thyroxine (T4) with a combination of T4 and triiodothyronine. J Clin Endocrinol Metab. 2007 Nov;92(11):4115-22

12. Jonklaas J. Update on the treatment of hypothyroidism. Curr Opin Oncol. 2016 Jan;28(1):18-25. doi: 10.1097/CCO.0000000000000242

13. Rajput R, Chatterjee S, Rajput M. Can levothyroxine be taken as evening dose? Comparative evaluation of morning versus evening dose of levothyroxine in treatment of hypothyroidism. J Thyroid Res. 2011;2011:505239.

14. Bensenor IM, Olmos RD, Lotufo PA. Hypothyroidism in the elderly: diagnosis and management. Clin Interv Aging. 2012;7:97-111.

15. Koulouri O, Auldin MA, Agarwal R, Kieffer V, Robertson C, Falconer Smith J, Levy MJ, Howlett TA. Diagnosis and treatment of hypothyroidism in TSH deficiency compared to primary thyroid disease: pituitary patients are at risk of under-replacement with levothyroxine. Clin Endocrinol. 2011;74:744-9.

16. Hirata Y, Fukuoka H, Iguchi G, Iwahashi Y, Fujita Y, Hari Y, Iga M, Nakajima S, Nishimoto Y, Mukai M, Hirota Y, Sakaguchi K, Ogawa W, Takahashi Y. Median-lower normal levels of serum thyroxine are associated with low triiodothyronine levels and body temperature in patients with central hypothyroidism. Eur J Endocrinol. 2015;173:247-56. doi: 10.1530/EJE-15-0130

Поступила 29.04.2019 